\title{
Effect of the mechanical stretching on the ferroelectric properties of a (VDF/TrFE) (75/25) copolymer film
}

\author{
V. Sencadas ${ }^{\mathrm{a}, \mathrm{b}}$, S. Lanceros-Méndez ${ }^{\mathrm{a}}$, J.F. Mano ${ }^{\mathrm{b}, \mathrm{c}, *}$ \\ ${ }^{a}$ Department of Physics, University of Minho, Campus de Gualtar, 4710-057 Braga, Portugal \\ ${ }^{\mathrm{b}}$ Department of Polymer Engineering, Universidade do Minho, Campus de Azurém, 4800-058 Guimarães, Portugal \\ '3B's Research Group-Biomaterials, Biodegradables and Biomimetics, University of Minho, Campus de Gualtar, $4710-057$ Braga, Portugal
}

Received 23 June 2003; accepted 18 July 2003 by H. Eschrig

\begin{abstract}
The transition phase behaviour of a vinylidene fluoride-trifluoroethylene (VDF-TrFE) copolymer film was studied after being subjected to different mechanical stretching levels in both longitudinal (L) and transversal (T) directions relatively to the initial extrusion direction. Both ferroelectric-paraelectric (FE-PE) and melting transitions were detected in the films by differential scanning calorimetry, that were not affected by $L$ stretching. This suggests that sliding mechanism along the $c$-axis of such films during plastic deformation influences slightly the all-trans chain conformations and the $\mathrm{CF}_{2}$ dipole orientation. On the other hand, the FE-PE peak decreases in area and temperature upon $T$ stretching, indicating a damage of the ferroelectric phase. However, the melting peak maintains unchanged. This fact provides evidence that the mechanical stretching do not change the total degree of crystallinity. The results suggest that the stretching induces a FE-PE phase transformation, without affecting the degree of crystallinity.
\end{abstract}

(C) 2003 Published by Elsevier Ltd.

PACS: 62.20. - x; 65.70. + y; 77.80. - e; 81.70. - q

Keywords: A. Ferroelectrics; A. Polymers; D. Mechanical properties; D. Thermodynamic properties

\section{Introduction}

Vinylidene fluoride-trifluoroethylene (VDF-TrFe) copolymers have attracted intense scientific and technological interest due to their unique ferroelectric phase transition within this family of compounds. This ferroelectric phase occurs in the bulk material at a transition temperature below melting $\left(T_{\mathrm{c}}<T_{\mathrm{m}}\right)$ without the need of additional electrical pooling or stretching. The crystalline structure in the ferroelectric phase is composed by all-trans chains with their dipoles parallel to the $b$-axis. Above $T_{\mathrm{c}}$, the paraelectric crystalline structure is hexagonal, essentially

\footnotetext{
* Corresponding author. Present address: Department of Polymer Engineering, Universidade do Minho, Campus de Azurém, 4800058 Guimarães, Portugal. Tel.: +351-253510320; fax: +351253510339

E-mail address: jmano@dep.uminho.pt (J.F. Mano).
}

consisting of a statistical combination of TT, TG and $\mathrm{TG}^{\prime}$ rotational isomers [1]. The Curie temperature is found to be highly dependent upon the VDF content [2,3]. Other factors also affect the location of this transition, such as heat treatments $[4,5]$, especially within the temperature range between $T_{\mathrm{c}}$ and the melt temperature, $T_{\mathrm{m}}$, or the effect of poling [6].

The disappearance of the FE phase above $T_{\mathrm{c}}$ involves drastic conformational changes from the trans to gauche states, accompanied by a rotational motion of the chains around the chain axis; in this case for copolymers with a VDF content higher than $70 \mathrm{~mol} \%$, this transition occurs in a reversible and discontinuous fashion [4].

Further, it was reported that $\mathrm{P}(\mathrm{VDF}-\mathrm{TrFE})$ after highenergy electron irradiation shows many features resembling those observed in relaxor ferroelectric systems [3]. This phenomenon was previously only observed in inorganic systems. 
Due to the interest in relating the changes in the nature and characteristics of the FE transition in this material to structural variations, in this work we have investigated the evolution of the ferroelectric-paraelectric (FE-PE) transition with increasing permanent (plastic) deformation along the two main directions of the material.

The slight anisotropic behaviour of P(VDF-TrFE) 75/25 observed in the stress-strain curves is reflected in the way the increasing deformation affects the transition, both in the behaviour of the transition temperature and in the shape and strength of the FE anomaly. The relationship between the structural changes, the local relaxation processes and the FE-PE transition gives us a better understanding of the FE behaviour at a microscopic scale in this class of materials.

This work also contributes to the investigation of the influence of the direction of the mechanical load in anisotropic films.

\section{Experimental}

Commercially available films of $\mathrm{P}(\mathrm{VDF}-\mathrm{TrFE})$ $75 / 25$ wt $\%$ were obtained from PIEZOTECH S.A., SaintLouis, France. The copolymer films with $100 \mu \mathrm{m}$ thickness were extruded and several $\mathrm{kV}$ were applied using the patented ISL-Buaer cycling process.

The morphology of the studied film was analysed by Xray diffraction using synchrotron radiation at the A2 Soft Condensed Matter Beamline of HASYLAB, DESY. 2D SAXS and WAXS patterns were simultaneously obtained, with the film aligned vertically along the $T$ direction. These measurements were performed at room temperature employing a MARCCD detector for the SAXS patterns and image plates as WAXS detectors, the sample-todetector distances being set to 180 and $22 \mathrm{~cm}$, respectively.

Tensile test were performed in a MINIMAT (Polymer Labs) machine in the tensile mode at test velocity of $\mathrm{d} l / \mathrm{d} t=2 \mathrm{~mm} / \mathrm{min}$ on $\mathrm{ca} .4 \mathrm{~cm} \times 1 \mathrm{~cm}$ rectangular samples in two different orthogonal directions. The orientation along the direction of the extrusion of the original film will be labelled $L$ (longitudinal), whereas the perpendicular orientation will be labelled $T$ (transversal). Films with different induced stretching levels, in the plastic region along both $T$ and $L$ directions, were prepared with the same equipment, by interrupting the tests at different strains.

Differential scanning calorimetry (DSC) experiments were performed with a Perkin-Elmer DSC7 apparatus. The temperature of the equipment was calibrated with indium and lead standards and for the heat flow calibration only the same indium sample was used. All experiments were carried out on heating at $10{ }^{\circ} \mathrm{C} \mathrm{min}^{-1}$. The samples for the DSC studies were cut from the middle region of the stretched films into small pieces and placed into $50 \mu \mathrm{l}$ pans. An effort was made to keep the same sample weight for the different films (typical $5.13 \pm 0.006 \mathrm{mg}$ ). These two procedures allowed to maintain as much as possible the same thermal resistance of the sample. This allowed to determine, in a comparative way, the transition temperatures $\left(T_{\mathrm{c}}\right.$ and $\left.T_{\mathrm{m}}\right)$, which were measured as the maximum of the peaks. Note that one should preferentially use the onset temperature of the peaks instead, but this was impossible for the case of $T_{\mathrm{m}}$ as the two peaks are partially overlapped.

\section{Results and discussion}

The representative quasi-static mechanical behaviour is presented in Fig. 1. For both directions a ductile behaviour is found, with a yield stress at ca. $35 \mathrm{MPa}$. The moduli measured in the two directions are almost the same (the tangent modulus at $2 \%$ is $0.73 \pm 0.1 \mathrm{GPa}$ for the $L$ direction and is $0.93 \pm 0.1 \mathrm{GPa}$ for $T$ direction). However, in the $T$ direction it is detected a more pronounced horizontal plastic behaviour, with a higher strain at break $(\sim 400 \%)$ than for the $L$ direction $(\sim 210 \%)$. In the former direction an initial localised deformation process lead to the formation of a neck. In the $L$ direction, the continuous increase in stress indicates a work hardening induced probably by the increase blocking of tied chains.

Those results point out for an anisotropic structure in the film, which was confirmed by X-ray diffraction experiments on the non-stretched film (Fig. 2). In fact, the (200) and (110) Bragg reflections at $2 \theta$ around $20^{\circ}$, the ones with higher contrast in the WAXS pattern (Fig. 2(a)), are more intense in the equatorial direction, indicating a higher crystalline orientation along the $L$ direction. Also the SAXS pattern evidences a lamellar structure that is preferentially oriented along the $L$ direction (Fig. 2(b)).

The piezoelectric property of (VDF-TrFE) copolymers

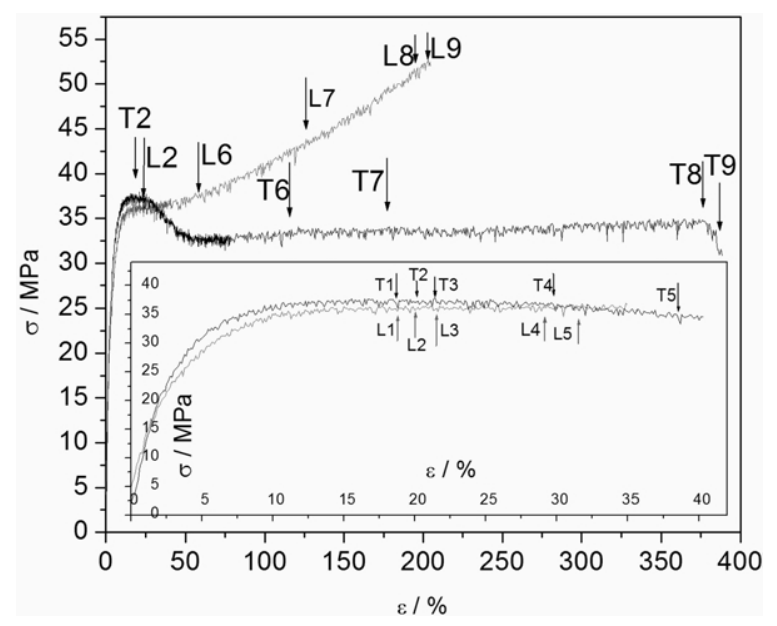

Fig. 1. Typical stress-strain curves of the studied VDF-TrFE copolymer performed in the two orthogonal (transverse) $T$ and (longitudinal) $L$ directions, relatively to the direction of the film processing. The inset graphic magnifies the behaviour up to $\varepsilon \sim$ $40 \%$. 


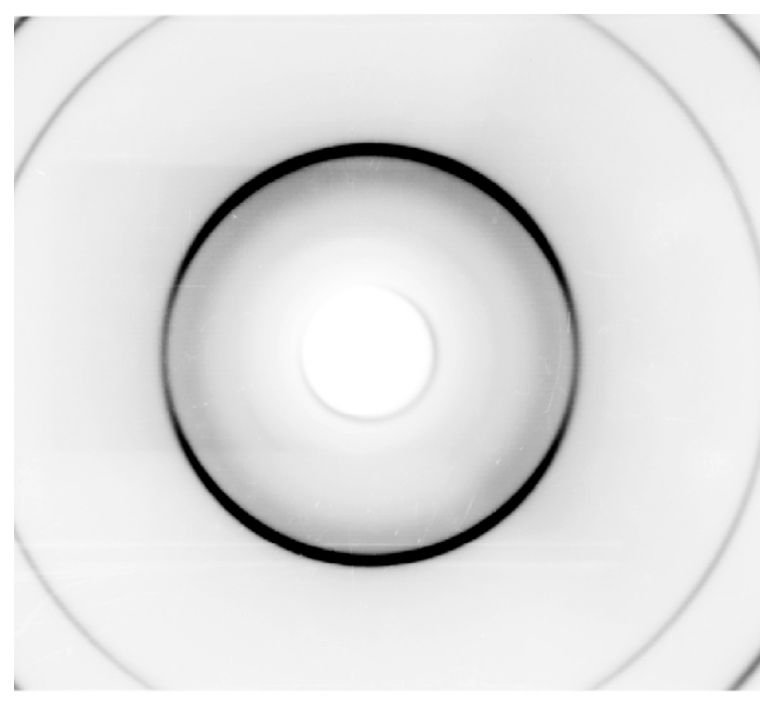

(a)

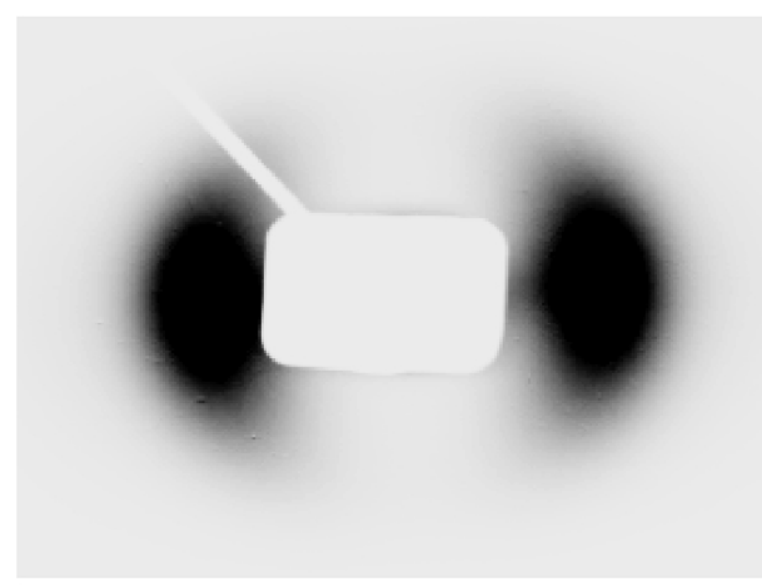

(b)

Fig. 2. 2D WAXS (a) and SAXS (b) patterns obtained in the original VDF/TrFE copolymer film.

is originated from the crystalline structure and the interface between this crystalline structure and the amorphous part. It is thus important to understand the behaviour and conformation of the crystalline part of the material under different conditions, in order to find strategies to obtain highly electroactive materials. During the processing of the material, it is known that drawing enhances chain orientation; also posterior annealing above $T_{\mathrm{c}}$ induces more perfect crystalline structures [4] and the effect of an electrical poling also produces higher degree of dipole orientation, i.e. higher piezoelectric response [6]. It would be interesting in this context to investigate the effect of the mechanical load in such systems, especially in their transition temperatures and structure.

Fig. 3 shows the DSC curves of the studied film after

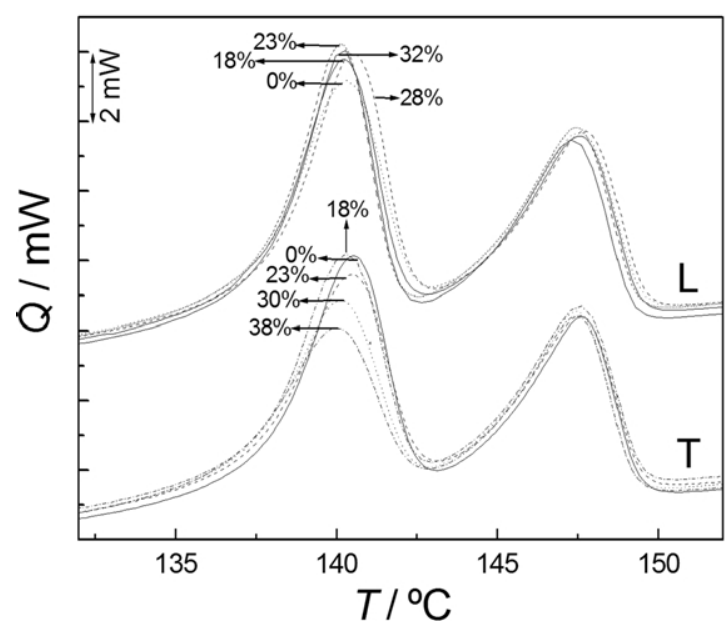

Fig. 3. DSC curves obtained at $10^{\circ} \mathrm{C} / \mathrm{min}$ on samples deformed along the $L$ (upper curves) and $T$ (bottom curves) directions, with different strain levels (indicated in the graphic).

different levels of irreversible stretching (beyond yielding) in both $L$ and $T$ directions. Two peaks are clearly observed, corresponding to the $\mathrm{FE}-\mathrm{PE}$ transition (Currie transition), with maxima at ca. $140{ }^{\circ} \mathrm{C}$, and to the melting of the paraelectric phase, located at ca. $147^{\circ} \mathrm{C}$. It can be observed in the original film that the area of both peaks are very similar. As this area is proportional to the transition enthalpy and the degree of crystallinity and considering that the whole crystalline part of the material is initially in the ferroelectric phase, the transition enthalpy of the two observed transitions must be similar. The crystalline structure of the original film is composed basically by ferroelectric phase and the crystalline part of the material undergoes a complete transformation from ferroelectric to paraelectric during the FE-PE transition.

For the stretching along the $L$ direction no significant changes are observed in both transitions up to $32 \%$ (transition temperature and enthalpy and broadness of the peaks). Fig. 4 shows the effect of stretching on both position and intensity of the FE-PE peak, including data obtained in higher deformed films. Only a slight decrease in the peak height is detected being its position unchanged upon stretching. This result indicates that the quality of the ferroelectric structure is maintained at high deformation levels induced by mechanical load along the $L$ direction, parallel to the $c$-axis of the crystalline structure. Further, the FE-PE transition is slightly stabilised, as demonstrated by the slight increase (however below the experimental error) of the transition temperature upon increasing deformation. Probably, only sliding between lamellae structures occurs, maintaining almost unchanged the all-trans chain conformations and the $\mathrm{CF}_{2}$ dipole orientation. More studies, using infrared spectroscopy and $\mathrm{X}$-ray diffraction are currently in progress, in order to confirm such hypothesis.

For the stretching along the $T$ direction systematic 

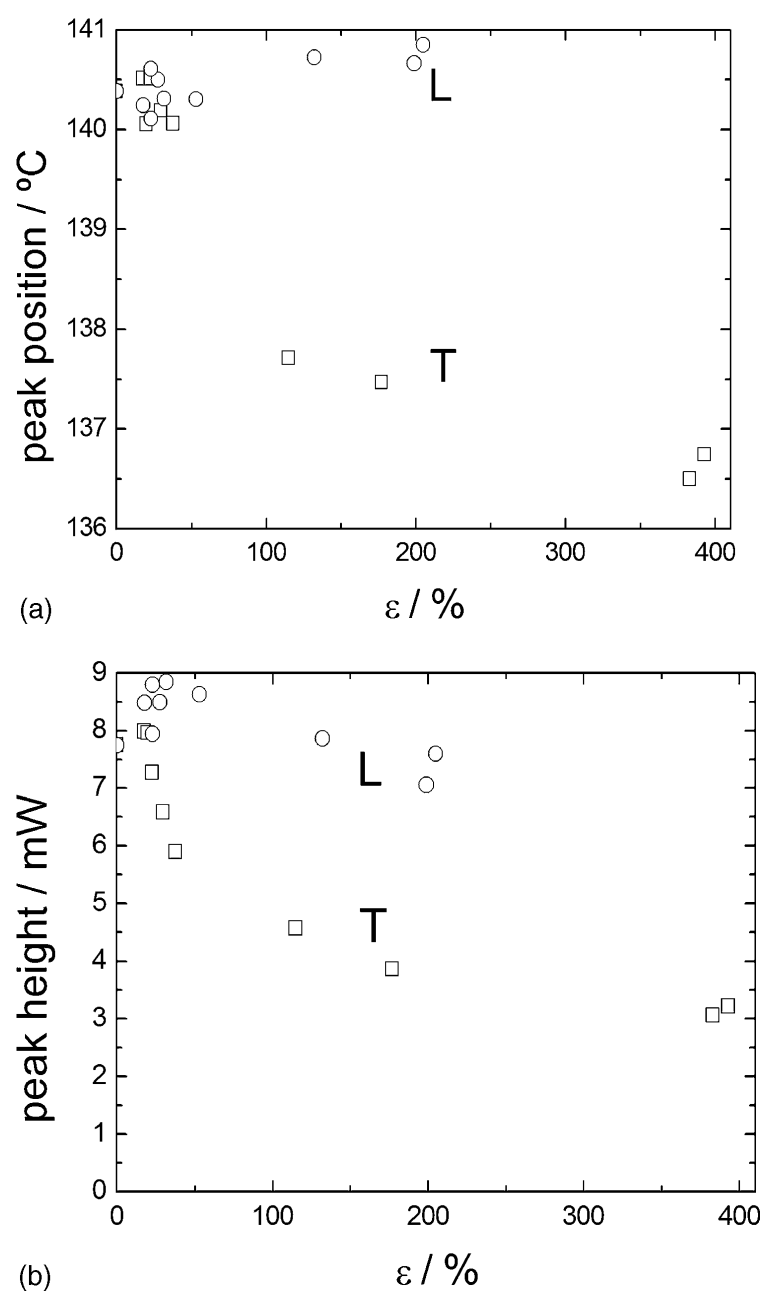

Fig. 4. Characteristics of the FE-PE transition peak observed by DSC for the sample deformed along the $L$ (circles) and $T$ (squares) as a function of the strain level. (a) Position of the peak in the temperature axis, (b) intensity of the peak in the heat flux axis, taking into account a linear base line.

changes are observed in the FE-PE peak (Fig. 3). The intensity (and thus the area) continuously decreases above the yielding point, indicating that the ferroelectric phase is disappearing during the deformation in this direction. Note that the height of the FE-PE peak for the broken film (strain of breaking at ca. $400 \%$ ) is about three times lower than the un-stretched film (Fig. 4(b)). Another consequence of this stretching is observed in the FE-PE peak position. Especially above $20 \%$ of deformation, the temperature of peak's maximum decreases strongly, occurring below $137^{\circ} \mathrm{C}$ at the breaking strain, i.e. a decrease in more than $3.5^{\circ} \mathrm{C}$ relatively to the pristine film. These facts suggest that the stretching along the $T$ direction degrades and destabilised the ferroelectric phase, mainly by reducing the lamellae thickness or by increasing of gauche defects (that also thermodynamically destabilises the ferroelectric phase), as the transition temperature decreases. The width at half maximum of the FE-PE peaks calculated from the DSC curves tends to increase as $\varepsilon$ increases. Therefore, de lamellae thinning or the increase in gauche defects is not uniform, but is rather represented by a more relative heterogeneous remaining crystalline phase, i.e. the average lamellae thickness decreases but its distribution broadens.

Interestingly, despite the changes in the FE-PE peak, almost no alterations are found in the melting peak with the stretching along the $T$ direction. Thus, the amount of paraelectric phase present just before the melting seems to be independent on the loss of ferroelectric phase due to the stretching. This suggests that this ferroelectric phase that is destroyed is transformed into a paraelectric phase, being the degree of crystallinity unaltered during the stretching process. The underlying mechanism may comprise melting and re-crystallisation of the chains removed from the ferroelectric phase, onto a new paraelectric phase, probably aligned along the stretched direction. Such change in orientation of the crystalline phase during plastic deformation, in films stretched perpendicular to the main film orientation, was found in pure PVDF analysed by FTIR [7, 8].

\section{Acknowledgements}

Work supported by the Portuguese Foundation for Science and Technology under Grant POCTI/CTM/33501/99. The X-ray experiments had the support of the HASYLAB Project II-01-006 EC.

\section{References}

[1] K. Tashiro, K. Takano, M. Kobayashi, H. Tadokoro, Polym. Commun. 22 (1981) 1312.

[2] H.S. Nalwa, Ferroelectric Polymers, Dekker, New York, 1995.

[3] V. Bharti, Q.M. Zhang, Phys. Rev. B 63 (2001) 184103.

[4] R. Tanaka, K. Tashiro, M. Kobayashi, Polymer 40 (1999) 3855.

[5] M.A. Barique, H. Ohigashi, Polymer 42 (2001) 4981.

[6] K.J. Kim, G.B. Kim, Polymer 38 (1997) 4881.

[7] S. Lanceros-Mendez, J.F. Mano, A.M. Costa, V.H. Schmidt, J. Macromol. Sci., Phys. B40 (2001) 517.

[8] S. Lanceros-Mendez, M.V. Moreira, J.F. Mano, V.H. Schmidt, G. Bohannan, Ferroelectrics 273 (2002) 2393. 